\title{
Effects of several quinones on insulin aggregation
}

SUBJECT AREAS: BIOPHYSICAL CHEMISTRY MOLECULAR BIOPHYSICS

Received

7 April 2014

Accepted

23 June 2014

Published

10 July 2014

Correspondence and requests for materials should be addressed to

L.Z. (lzheng217@

hotmail.com) or K.H.

(kunhuang2008@

hotmail.com)

\author{
Hao Gong' , Zihao He' , Anlin Peng' ${ }^{2}$, Xin Zhang' ', Biao Cheng' ', Yue Sun ${ }^{3}$, Ling Zheng ${ }^{3}$ \& Kun Huang 1,4
}

${ }^{1}$ Tongji School of Pharmacy, Huazhong University of Science and Technology, Wuhan, Hubei, P. R. China430030, ${ }^{2}$ Department of Pharmacy, The Third Hospital of Wuhan, Wuhan, Hubei, P. R. China430060, ${ }^{3}$ College of Life Sciences, Wuhan University, Wuhan, Hubei, P. R. China430072, ${ }^{4}$ Centre for Biomedicine Research, Wuhan Institute of Biotechnology, Wuhan, Hubei, P. R. China430075.

Protein misfolding and aggregation are associated with more than twenty diseases, such as neurodegenerative diseases and metabolic diseases. The amyloid oligomers and fibrils may induce cell membrane disruption and lead to cell apoptosis. A great number of studies have focused on discovery of amyloid inhibitors which may prevent or treat amyloidosis diseases. Polyphenols have been extensively studied as a class of amyloid inhibitors, with several polyphenols under clinical trials as anti-neurodegenerative drugs. As oxidative intermediates of natural polyphenols, quinones widely exist in medicinal plants or food. In this study, we used insulin as an amyloid model to test the anti-amyloid effects of four simple quinones and four natural anthraquinone derivatives from rhubarb, a traditional herbal medicine used for treating Alzheimer's disease. Our results demonstrated that all eight quinones show inhibitory effects to different extent on insulin oligomerization, especially for 1,4-benzoquinone and 1,4-naphthoquinone. Significantly attenuated oligomerization, reduced amount of amyloid fibrils and reduced hemolysis levels were found after quinones treatments, indicating quinones may inhibit insulin from forming toxic oligomeric species. The results suggest a potential action of native anthraquinone derivatives in preventing protein misfolding diseases, the quinone skeleton may thus be further explored for designing effective anti-amyloidosis compounds.

myloid formation plays an important role in the pathogenesis of misfolding-based diseases such as Parkinson's disease, Alzheimer's disease and type 2 diabetes mellitus $^{1-3}$. Under appropriate condition, proteins may form toxic aggregates by first misfolding into $\beta$-sheet-rich intermediates, which further assemble into soluble oligomers and finally to linear mature fibrils. The aggregated species formed in this process may attach to cell membranes and cause membrane permeabilization, which induce cell dysfunction and apoptosis ${ }^{5,6}$. It is generally believed that interruption of amyloid aggregation may provide a plausible therapeutic approach for protein misfolding diseases ${ }^{7}$.

Insulin is a hypoglycemic hormone that widely used as anti-diabetic drug. Insulin amyloidosis was commonly found at the site of repeated insulin injection in diabetic patients as well as in the clinic formulations ${ }^{8-11}$. Different from the natively unstructured amyloidogenic proteins such as islet amyloid polypeptide, amyloid- $\beta$ and $\alpha$ synuclein ${ }^{12-14}$, insulin belongs to the category of natively structured amyloidogenic proteins, such as prion and lysozyme $e^{15-17}$, and is also commonly used as a classical model to probe the mechanisms of protein fibrillation since insulin amyloids share the same typical cross- $\beta$ structure as the mature fibrils of the disease relevant amyloids ${ }^{4,18,19}$, as well as an amyloid model system for studying aggregation inhibitors ${ }^{20,21}$.

Many aggregation inhibitors have been identified to affect aggregation process, for example, small molecules, peptides and oligomer- or amyloid-specific antibodies ${ }^{22}$. Among them, native polyphenols from food or herbal medicines, such as epigallocatechin gallate (EGCG), curcumin and resveratrol, have been well-studied as amyloid inhibitors $^{23-25}$. The tea catechin polyphenol, EGCG, can inhibit the aggregation of various proteins and is being assessed in clinical trials for Alzheimer's disease ${ }^{26}$. Quinones, the oxidation products of polyphenols that include benzoquinones $(\mathrm{BQ})$, naphthoquinones $(\mathrm{NQ})$, anthraquinones $(\mathrm{AQ})$ and phenanthraquinones $(\mathrm{PQ})$, are widely found in nature. Recently, several quinones have been reported to inhibit amyloid aggregation efficiently. For example, 1,4-benzoquinone, the simplest quinone, inhibits lysozyme fibrillization more efficiently than the corresponding phenol $^{27}$. Similarly, 9,10-anthraquinone strongly inhibits the aggregation of $\beta$-amyloid protein ${ }^{28}$. In addition, several derivatives of 1,4-naphthoquinone have been explored as drug candidates for amyloidosis diseases $^{29}$. These evidences all implicate quinones as a class of compounds with inhibitory effect on protein misfolding and aggregation. 
A number of bioactive derivatives of AQ can be found in rhubarb extract (Rheum rhabarbarum L.), a traditional herbal medicine which may be used for treating amyloidosis related diseases such as Alzheimer's disease, with a possible mechanism that some of its effective components may affect $\beta$-amyloid protein related pathogenic pathways ${ }^{30,31}$. One of the anthraquinones, emodin (EM), has been reported to effectively inhibit tau (a microtubule-associated protein) aggregation and show neuroprotective effect ${ }^{32}$. Previous reports on natural quinones mainly focus on the mechanisms behind their anti-cancer or anti-bacterial activities ${ }^{33,34}$, their exact effects and mechanisms on protein misfolding diseases remain limited. Here, we propose that rhubarb anthraquinones may affect protein misfolding and aggregation. To test this hypothesis, the amyloid inhibitory effects of a set of simple quinones and natural AQ derivatives from rhubarb (Figure 1) were compared, with insulin used as a model amyloid system.

\section{Results}

Quinones inhibit amyloid formation of insulin. Thioflavin-T (ThT) based fluorescence was used to monitor the aggregation process of $100 \mu \mathrm{M}$ insulin that co-incubated with $50 \mu \mathrm{M}$ of various quinones at $65^{\circ} \mathrm{C}$. The morphology of insulin aggregates in the absence or presence of quinones was observed by transmission electronic microscopy (TEM). Control insulin gave a strong ThT emission with a lag time of $4.52 \pm 0.45 \mathrm{~h}$ and reached a plateau within $14 \mathrm{~h}$ (Figure 2A). After incubation for $24 \mathrm{~h}$, TEM image showed a mesh of insulin aggregates characterized as linear fibrils (Figure 3A). Except AQ, the other three simple quinones all prolonged the aggregation lag time to different extent. BQ and NQ showed the strongest inhibitory effect accompanied with significantly decreased fluorescence intensities (Figure 2A), consistently, only small dispersed amorphous aggregates were identified in TEM (Figure 3B \& 3C). In contrast, PQ (9,10-phenanthraquinone) treatment prolongs the lag time of insulin to $6.97 \pm 0.48 \mathrm{~h}$ $(\mathrm{P}<0.05)$, but the maximum fluorescence intensity after $24 \mathrm{~h}$ incubation was similar to that of insulin alone (Figure 2A).
Although AQ did not extend the lag time $(4.51 \pm 0.23 \mathrm{~h})$, it nevertheless significantly reduced the maximum fluorescence intensity by $40 \%$ (Figure $2 \mathrm{~A}$ ). Despite the detectable increased fluorescence intensity after prolonged incubation, classic fibrils were not found in samples co-incubated with AQ and PQ, only scattered amorphous aggregates were observed (Figure 3D \& 3E) similar to a previous report ${ }^{35}$.

Compared to AQ, four AQ derivatives from rhubarb showed no significant effects on lag time; however, slightly stronger inhibitory effects in terms of maximum fluorescence intensities were observed (Table 1), with CP (chrysophanol) and EM showed the greatest inhibition effects (Figure 2B). Large amount of amorphous aggregates was observed by TEM for samples co-incubated with the rhubarb anthraquinone derivatives (Figure 3F-3I); however, for AE (aloe-emodin) and and RH (rhein), tiny short protofibril-like structures were found buried in the amorphous aggregates (Figure 3H \& 3I, enlarged frames).

To investigate whether quinones can affect the aggregation of already formed amyloid intermediates, each quinone was added to pre-incubated insulin (9 h). BQ and NQ suppressed the increase of fluorescence intensity in the following incubation, indicating blockage to the aggregation of already formed amyloid intermediates (Figure 2C). In contrast, PQ and AQ derivatives showed little inhibitory effects to pre-formed amyloid intermediates since the fluorescence continued to increase (Figure 2C). We further studied whether quinones can disaggregate preformed fibrils, quinones were added to insulin amyloids pre-incubated for $27 \mathrm{~h}$. Partial disaggregation effects were detected in BQ and NQ treated samples, while no disaggregation effects were found in all other samples according to ThT fluorescence assays (Figure 2C).

Quinones delay the secondary structural transformation of insulin. Far-UV CD spectroscopy was applied to analyze the effects of quinones on the secondary structure transition of insulin during amyloid formation. For native insulin, which is a highly structured molecule rich in $\alpha$-helix, two characteristic minima at 208 and $222 \mathrm{~nm}$ were

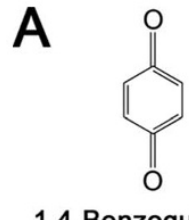

1,4-Benzoquinone (BQ)

B<smiles>Cc1cc(O)c2c(c1)C(=O)c1cccc(O)c1C2=O</smiles>
(CP)<smiles>CC(C)OC(=O)OCc1ccc(CO)cc1C(=O)OCc1ccccc1</smiles>
(AE)<smiles>O=C1c2ccccc2C(=O)c2ccccc21</smiles>

9,10-Anthraquinone (AQ)<smiles>Cc1cc(O)c2c(c1)C(=O)c1c(O)cc(O)cc1C2=O</smiles><smiles>[R][R]([R])([R])C</smiles>

Figure 1 Chemical structures of quinones investigated in this study. (A) Simple quinones: 1,4-benzoquinone (BQ), 1,4-naphthoquinone (NQ), 9,10-anthraquinone (AQ) and 9,10-phenanthraquinone (PQ); (B) Anthraquinone derivatives from rhubarb: chrysophanol (CP), emodin (EM), aloe-emodin (AE) and rhein (RH). 

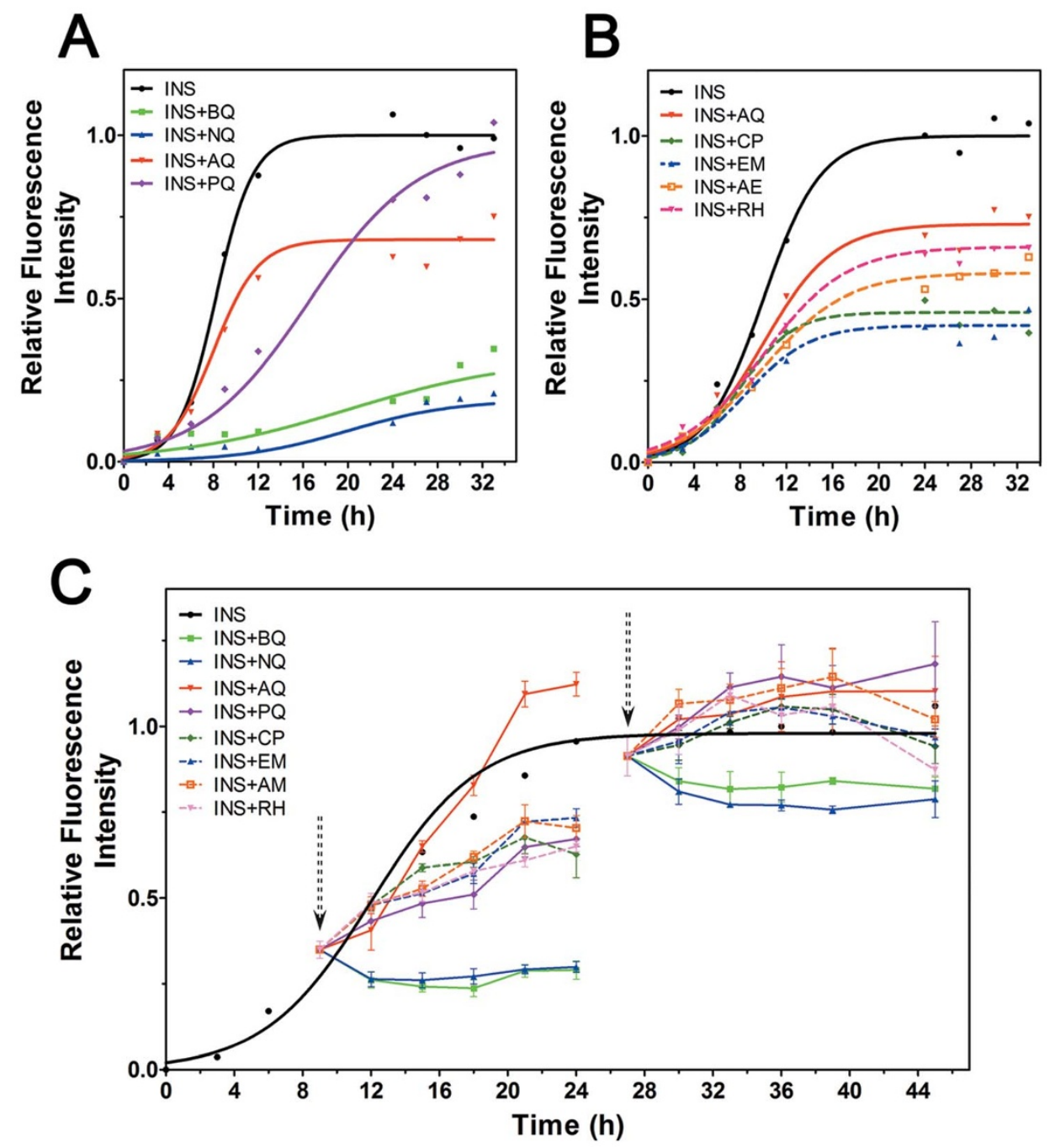

Figure $2 \mid$ Effects of quinones on amyloid formation of insulin. (A) Relative ThT fluorescence of insulin in the presence or absence of simple quinones; (B) Relative ThT fluorescence of insulin in the presence or absence of anthraquinone derivatives from rhubarb, AQ was used as a control; (C) Relative ThT fluorescence of pre-incubated insulin with quinones treatments start at $9 \mathrm{~h}$ or $27 \mathrm{~h}$, the arrows indicate the quinones adding points.

observed in CD spectrum (Figure 4A). During incubation, these two minima were decreased, indicating decreased content of $\alpha$-helix (Figure 4A). The incubation also caused reduced ellipticity near $200 \mathrm{~nm}$ that represents formation of random coil conformation during incubation (Figure 4A), which is similar to a previous research studied under extreme temperatures ${ }^{36}$. $\beta$-structure formation is regarded as a generic structural conversion in protein aggregation ${ }^{37}$, typical $\beta$-structure signature at $215 \mathrm{~nm}$ was not obvious until the fluorescence intensity of insulin reached its maximum at $24 \mathrm{~h}$.

For quinones treated samples, it was interesting to note that the addition of quinones immediately decreased the positive signal at $200 \mathrm{~nm}$ and increased the negative band near $220 \mathrm{~nm}$ which refer to a reduction of $\alpha$-helix content (Figure 4A \& 4B), suggesting there may be a direct interaction between quinones and insulin. After coincubated with quinones for $6 \mathrm{~h}$ and $12 \mathrm{~h}$, the spectral changes at 200 and $220 \mathrm{~nm}$ were significantly less compared to those of the insulin control, indicating quinones delay the structural transformation (Figure 4F \& 4G), which was consistent with the accompanying ThT-fluorescence measurements (Figure 4C \& 4D). At the end of $24 \mathrm{~h}$ incubation, although the fluorescence still showed notable inhibitory effects of quinones (Figure $4 \mathrm{E}$ ), the CD spectra nevertheless suggested the secondary structures of all quinones treated samples were similar to that of control insulin (Figure $4 \mathrm{H}$ ).
Quinones inhibit the oligomerization and fibrillation of insulin. Size-exclusion chromatography (SEC) was first used to analyze the oligomerization states. For insulin, the monomer showed a single peak eluting at $22.4 \mathrm{~min}$ with a calculated molecular weight (MW) of $5.8 \mathrm{kD}$, while the addition of quinones caused no change to the monomeric status of insulin at the beginning of the incubation (Figure 5 \& Figure S1). After incubating for $6 \mathrm{~h}$, small peaks appeared with elution time between $19.5-21.5 \mathrm{~min}(\mathrm{ca} .10-16 \mathrm{kD})$, suggesting the formation of dimers and trimers (Figure 5). The intensities of these dimer and trimer peaks rose sharply after $12 \mathrm{~h}$ incubation (Figure 5), whereas quinones strongly inhibited the oligomerization of insulin (Figure 5 \& Figure S2). This result agrees with those obtained from the CD spectrometry and ThT fluorescence assay, which was further corroborated by an immuno dot-blot assay, which probes the formation of oligomers and fibrils in the incubated samples with the oligomer- or fibril-specific antibodies (Figure S3).

Dynamic light scattering (DLS) measurements were further performed to monitor particle size distributions (Figure 6). At $0 \mathrm{~h}$, the average diameter of each sample was less than $100 \mathrm{~nm}$ (data not shown). For insulin, oligomers with diameters over $1000 \mathrm{~nm}$ were observed after $8 \mathrm{~h}$ incubation, and fibrils with size greater than $5000 \mathrm{~nm}$ were detected after $30 \mathrm{~h}$. In contrast, at each time point, 


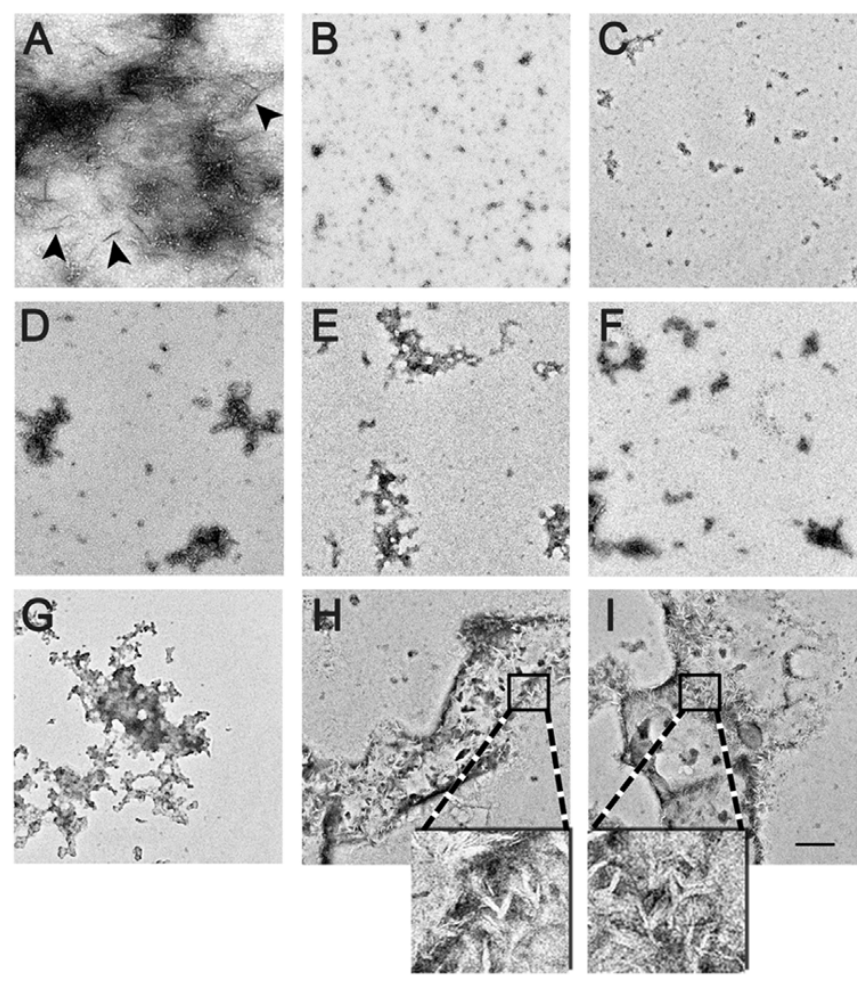

Figure 3 TEM images of amyloid formed in different sample groups. (A) Insulin; (B)-(I) insulin co-incubated with BQ, NQ, AQ, PQ, CP, EM, $\mathrm{AE}, \mathrm{RH}$, respectively. The enlarged frames $(4 \mathrm{x})$ show details of tiny protofibril-like structures buried in the amorphous aggregates. Scale bar represents $200 \mathrm{~nm}$.

the average particle sizes of all quinones treated samples were less than those of insulin alone, with BQ- and NQ-treatment showed the strongest effects in reducing the average particle sizes of insulin. Consistent with the ThT results, rhubarb anthraquinone derivatives also showed inhibitory effects greater than that of AQ (Figure 6). Among all eight quinones tested, NQ has the highest inhibitory effect as shown in a colour map (Figure 6).

Quinones attenuate membrane disruption effects that induced by toxic insulin aggregates. Oligomers and mature amyloids have been shown to disintegrate cell membrane and cause cell death ${ }^{38,39}$. Erythrocyte and insulin aggregates were co-incubated to simulate cell membrane disruption. After $24 \mathrm{~h}$ incubation, insulin fibrils induced a hemolysis rate of $57.5 \pm 2.5 \%$, which was higher than that of PBStreated controls $(33.6 \pm 8.3 \%, \mathrm{P}<0.05)$. In quinones-treated samples, all hemolysis rates were decreased to $c a$. $40 \%$ of that of insulin $(\mathrm{P}<0.05$; Figure $7 \mathrm{~A})$. To investigate whether quinones by themselves interrupt the binding between insulin and membrane, a control study was performed in which quinones were added to completely formed insulin fibrils, and then mixed with erythrocyte for further incubation, the results showed quinones could not attenuate the membrane disruption effect of insulin fibrils except for RH (Figure 7B). Another control study suggested quinones by themselves had no protective effect on erythrocyte hemolysis, some compounds even slightly induced hemolysis (Figure S4). Unlike ThT data, no difference was found between simple quinones and rhubarb anthraquinone derivatives comparing their anti membrane disruption effects.

\section{Discussion and Conclusion}

According to classic amyloidogenesis model, insulin amyloid formation may be divided into two stages, in the initial oligomerization
Table 1 | Effects of quinones on the amyloidogenecity of insulin

\begin{tabular}{|c|c|c|c|}
\hline & Lag time (h) & $T_{50}(h)$ & Maximum intensity ${ }^{[2]}$ \\
\hline INS ${ }^{[1]}$ & $4.52 \pm 0.45$ & $8.17 \pm 0.85$ & $1.04 \pm 0.05$ \\
\hline $\mathrm{INS}+\mathrm{BQ}$ & N.D. ${ }^{[3]}$ & N.D. & $0.19 \pm 0.04$ \\
\hline $\mathrm{INS}+\mathrm{NQ}$ & N.D. & N.D. & $0.12 \pm 0.02$ \\
\hline $\mathrm{INS}+\mathrm{AQ}$ & $4.51 \pm 0.23$ & $7.83 \pm 1.03$ & $0.63 \pm 0.04$ \\
\hline INS + PQ & $6.97 \pm 0.48$ & $13.00 \pm 0.82$ & $0.77 \pm 0.06$ \\
\hline INS + CP & $5.01 \pm 0.32$ & $8.50 \pm 0.41$ & $0.50 \pm 0.09$ \\
\hline INS + EM & $4.73 \pm 0.27$ & $8.67 \pm 0.94$ & $0.42 \pm 0.05$ \\
\hline $\mathrm{INS}+\mathrm{AE}$ & $4.67 \pm 0.54$ & $9.67 \pm 1.70$ & $0.50 \pm 0.19$ \\
\hline INS + RH & $4.75 \pm 0.43$ & $9.45 \pm 0.43$ & $0.64 \pm 0.10$ \\
\hline
\end{tabular}

stage, insulin monomers misfold and assemble into small molecular weight oligomers including dimers, trimers and other low MW oligomers; in the second fibrillization stage, low order oligomers assemble into large oligomers which grow into linear fibrils ${ }^{40,41}$ (Figure 8). Recent studies also reported the existence of "offpathway" oligomerization pathways during protein misfolding ${ }^{42}$ (Figure 8). For example, it was revealed that $\alpha$-synuclein (an amyloidogenic protein associated with Parkinson's disease) may form nontoxic and unstructured non-amyloid aggregates ${ }^{43}$. In another elegant recent study, EGCG was found to redirect the amyloid formation of $A \beta$ into off-pathway nontoxic aggregates ${ }^{44}$. Here, we found all eight tested quinones inhibit the classic amyloid formation pathways of insulin, both in the oligomerization and fibrillization stages. Since for the samples treated with AQ, PQ and AQ derivatives all showed un-classic fibrillation characters including the complete or partial formation of amorphous aggregates that unlike typical linear amyloid morphology, decreased ThT fluorescence intensities, decreased average particle sizes as detected by DLS, and significantly attenuated toxicities as the hemolysis assays indicated. All these phenomena suggested that quinones may drive insulin undergoing the "off-pathway" aggregation while inhibiting the classic amyloid formation pathway (Figure 8). Although the exact mechanisms of quinones' inhibitory effects remain unclear, we reason that quinones may directly interact with insulin as the $\mathrm{CD}$ and hemolysis results suggested and thus disrupt oligomerization, similar to what EGCG does to the amyloid formation of $A \beta$ and $\alpha$-synuclein ${ }^{43,44}$. It will be of great future interest to further probe the mechanism with the nuclear magnetic resonance (NMR) spectrometry and molecular dynamics simulation approaches, which have been successfully applied in mapping the interaction sites between amyloidogenic proteins and small ligands ${ }^{45,46}$, and also in studying insulin and monosaccharides interaction $^{47}$.

It is worth mentioning that BQ and NQ, two simplest quinones used in this study, showed the highest efficacy in inhibiting insulin aggregation; whereas $\mathrm{AQ}$ and $\mathrm{PQ}$, which containing more benzene rings, showed lower inhibitory effects. The amyloid inhibitory effects of the eight quinones follow the rank order of NQ $>\mathrm{BQ}>\mathrm{PQ}>\mathrm{EM}$ $>\mathrm{CP}>\mathrm{AE}>\mathrm{RH}>\mathrm{AQ}$. It is generally accepted that aromaticintereactions and hydrophobic interactions are two major known driving forces responsible for amyloid formation ${ }^{48,49}$. For quinones, molecular simulations have implicated that quinones may destabilize the intermolecular cross- $\beta$ strand hydrogen bonds of oligomers and mature fibrils via more favorable polar interactions with the protein backbones, the quinonic moiety may bind to peptide backbone carbonyl oxygens and amide hydrogens tightly through $\pi^{+} \delta^{-}$interactions and hydrogen bonds, which allow the intercalation of the quinone molecule into the protein oligomers ${ }^{28,50}$. We speculate that the aromatic ring of monocyclic and dicyclic quinones (BQ and NQ) may also competitively interact with aromatic residues and thus intercalate into quino-protein oligomers which can not further ag- 

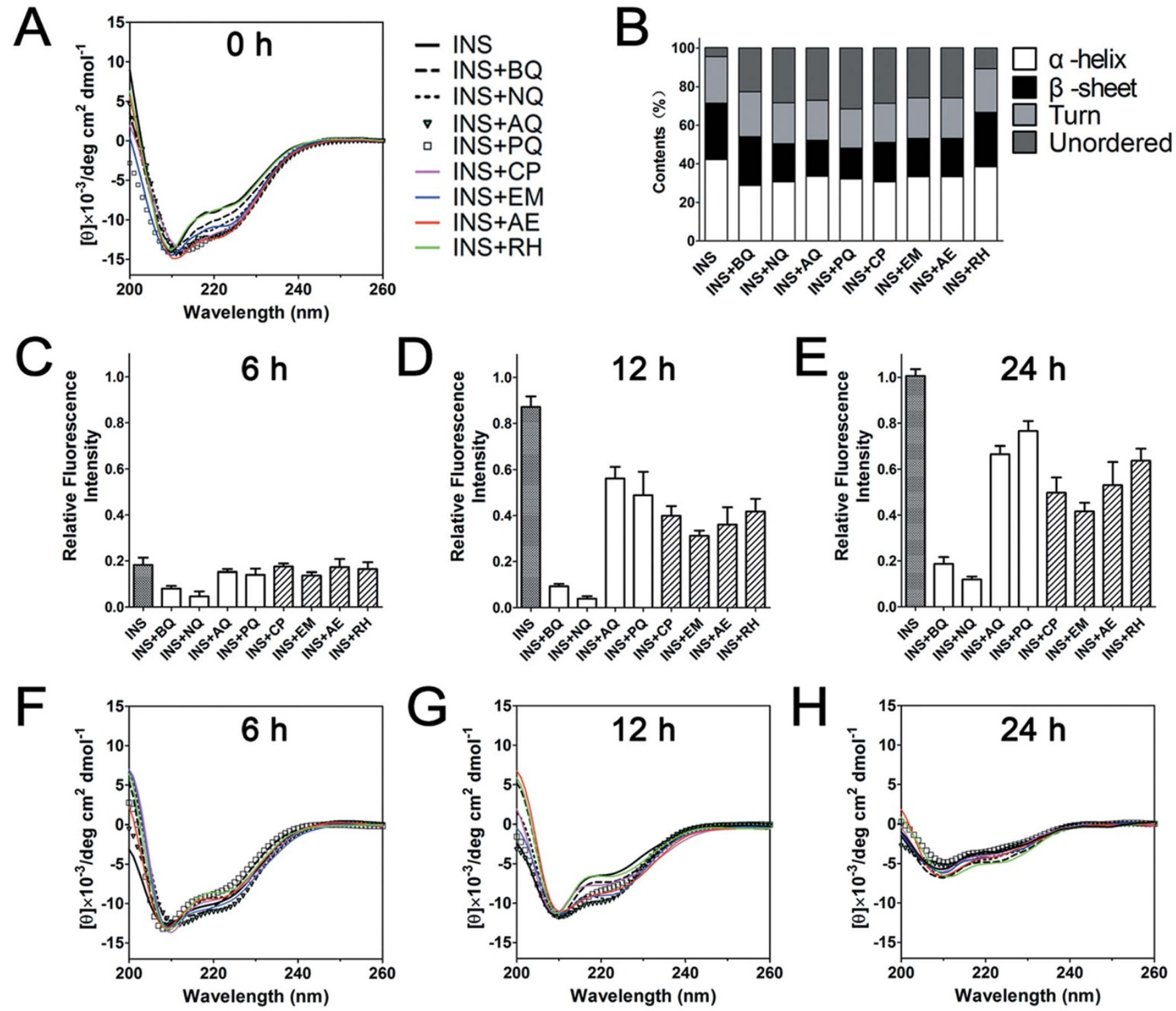

Figure $4 \mid$ Secondary structures of insulin in the presence and absence of quinones. Far-UV circular dichroism spectra of sample groups at $0 \mathrm{~h}$ (A), and percentages of secondary structure contents (B). Relative thioflavin-T fluorescence intensities at $6 \mathrm{~h}(\mathrm{C}), 12 \mathrm{~h}(\mathrm{D}) .24 \mathrm{~h}(\mathrm{E})$ and corresponding far-UV circular dichroism spectra (F-H). The fluorescence signals of all samples were normalized against that of insulin incubated for $24 \mathrm{~h}$, which was set as 1 .

gregate into fibrils. For tricyclic quinones, the extra benzene ring may increase the steric hindrance of the molecule and interfere the binding of compound and protein. For rhubarb derived AQ derivatives, the inhibitory effect may be influenced by substituent groups. It has been reported that aromatic amyloid inhibitors with strong electron withdrawing group on monocyclic benzene may increase the inhibitory potency ${ }^{51}$. For tri- and tetra-substituted AQ with more than two electron donating phenolic hydroxyl groups, the regulator may be more complex than that of monocyclic compounds. The phenolic hydroxyl groups may bind with hydrophobic residues to prevent hydrophobic interaction caused oligomerization, which may explain why AQ derivatives inhibit aggregation more efficiently than AQ. In addition, different factors such as metals and peptides may also affect the aggregation properties ${ }^{52,53}$, several small molecules have been reported to inhibit amyloid aggregation by chelating metal ions from metal-A $\beta$ species $^{54,55}$. Since quinones and their derivatives have also been reported as chelators ${ }^{56}$, it will be interesting to investigate whether quinones can also interrupt the ligands (such as metal ions) induced misfolding and aggregation.
In $\beta$-amyloid model, natural quinones such as pyrroloquinoline quinone and $\alpha$-tocopherol quinone were reported as effective amyloid inhibitors on aggregation ${ }^{35,57}$. Simple quinones in our study, including the first time studied 9,10-phenanthraquinone, may act as functional skeleton entities that contribute to the inhibitory effect of natural quinones. Moreover, anthraquinone derivatives from $r h u$ barb further indicate that natural quinones with similar configuration may provide a huge resource of amyloid inhibitors. Although some quinones from plants have been thought to be allergens ${ }^{58}$, quinones are still important sources for deriving new anti-amyloid inhibitors. And more extensive conditions such as metal ions,crowding, pressure and shear force may be considered to validate the present results. It will also be interesting to extent this research to traditional natural medicines containing more complex quinones as well as to other pathogenic amyloid systems.

\section{Methods}

Materials. Porcine insulin was obtained from Wanbang Biopharmaceutics INC. (Xuzhou, China). 1,4-benzoquinone, 1,4-naphthoquinone, 9,10-anthraquinone, 


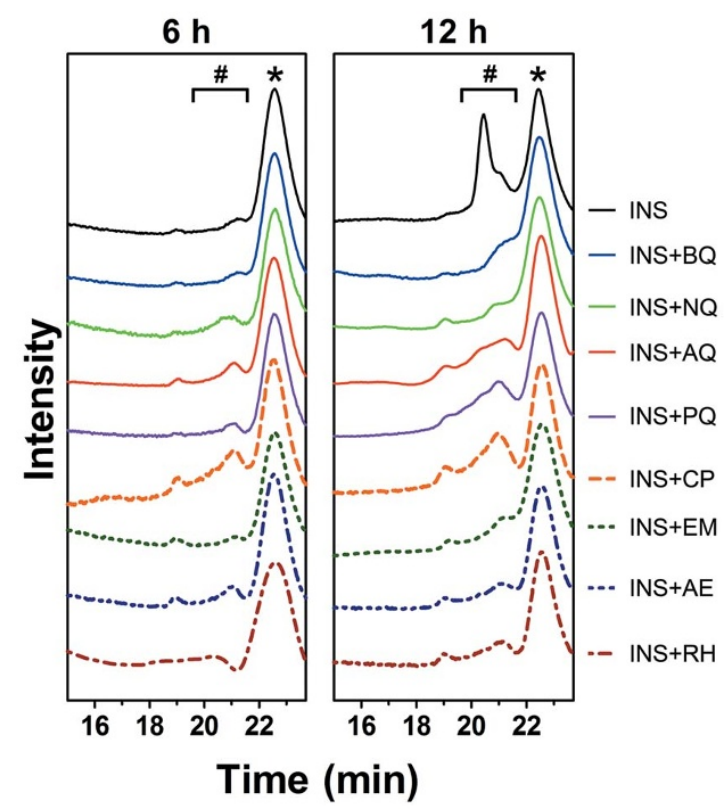

Figure 5 | Size-exclusion gel filtration profiles of insulin co-incubated with quinones. ${ }^{*}$ insulin monomers; ${ }^{*}$ insulin oligomers.

9,10-phenanthraquinone, chrysophanol (1,8-dihydroxy-3-methyl-anthraquinone), emodin (1,3,8-Trihydroxy-6- methyl-anthraquinone), aloe-emodin (1,8-dihydroxy3-hydroxymethyl- anthraquinone) and rhein (1,8-dihydroxy-3-carboxyanthraxquinone) were obtained from Aladdin-Reagents (Shanghai, China). Thioflavin-T (ThT) was from Sigma-Aldrich (St. Louis, USA). Anti-oligomer antibody (A-11), anti-fibril antibody (OC) and anti-rabbit IgG were from Merck Millipore (Billerica, USA). All other chemicals were of the highest grade available. For hemolytic assays, fresh blood was collected from healthy volunteers with approval by the ethics committees of the Third Hospital of Wuhan.

Amyloid formation and thioflavin-T (ThT) fluorescence assays. Insulin was dissolved in $50 \mathrm{mM}$ PBS (100 mM NaCl, pH 7.4) to a final concentration of $100 \mu \mathrm{M}$. Freshly prepared solution was mixed with different quinones in a molar ratio of protein:compound $=2: 1$ and sonicated for $2 \mathrm{~min}$. DMSO was used to prepare the stock solutions of quinones with a final solution DMSO concentration of $0.5 \%$ (control study demonstrated that 0.5\% DMSO has no significant effect on aggregation, data not shown). All groups were static incubated at $65^{\circ} \mathrm{C}$ to form aggregates. The aggregation status of designated time points was determined by ThT

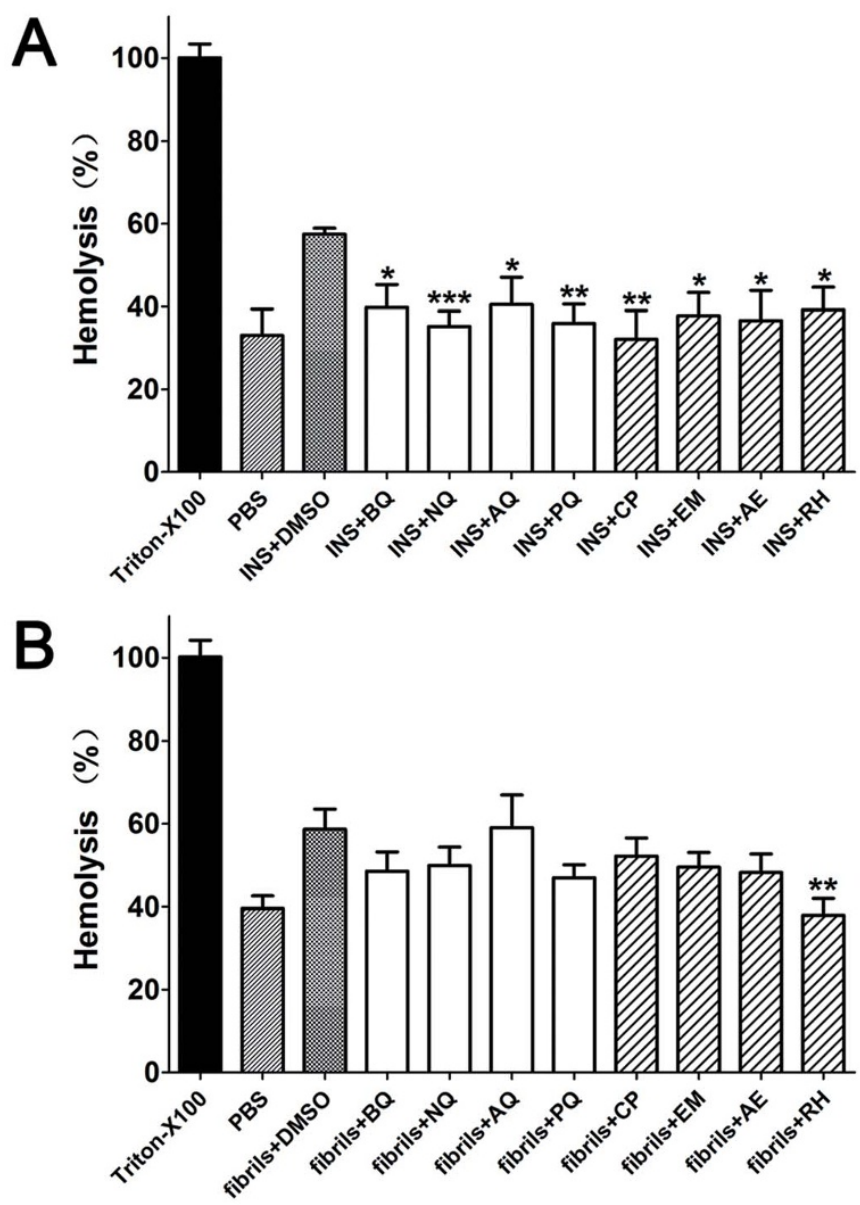

Figure $7 \mid$ The hemolysis rates of insulin aggregates in the presence or absence of quinones. (A) Hemolysis levels of pre-prepared quinonescoincubated insulin solution; (B) Hemolysis rates of already formed insulin fibrils with quinones added then. *, $\mathrm{P}<0.05$; **, $\mathrm{P}<0.01$ and $* * *, \mathrm{P}<0.001$ compared with the group of protein with DMSO.
$8 \mathrm{~h}$

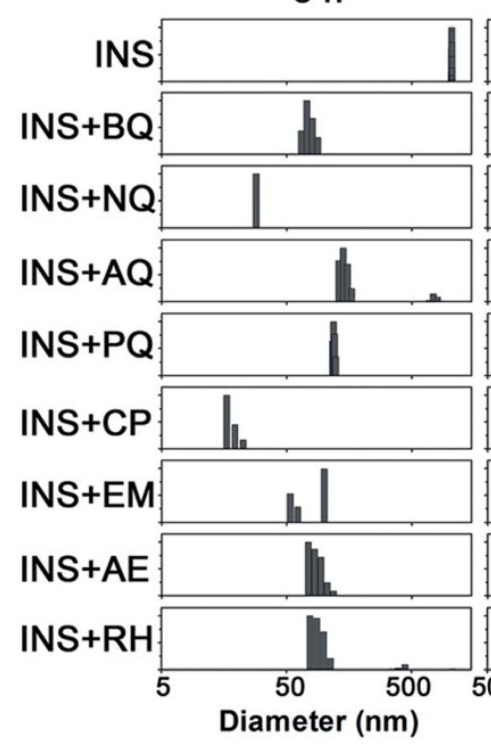

$15 \mathrm{~h}$
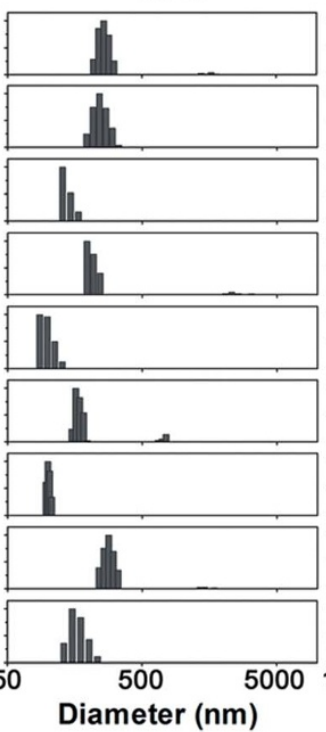

$30 \mathrm{~h}$

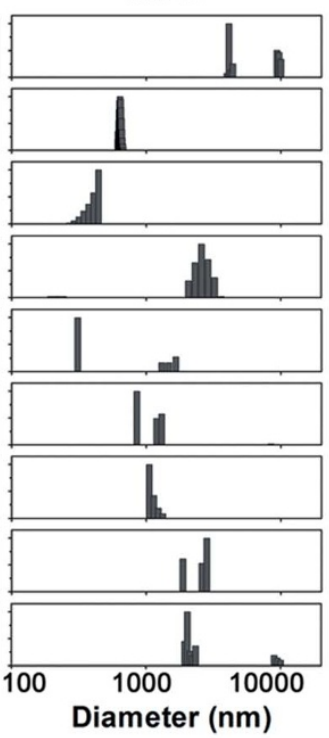

$0 \mathrm{~h} 8 \mathrm{~h} 15 \mathrm{~h} 30 \mathrm{~h}$

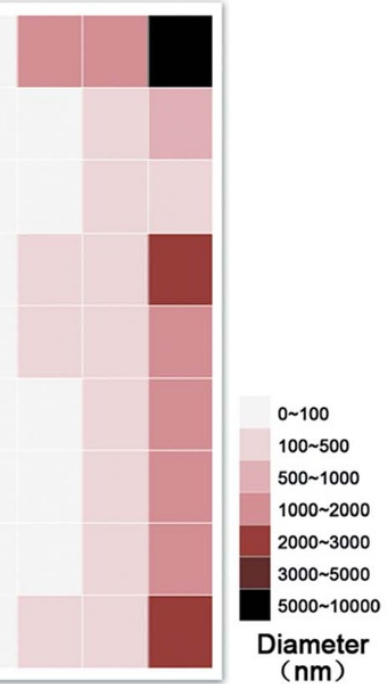

Figure 6 | Particle size distributions detected by dynamic light scattering. The colour map on the right summarized the size distributions of all experimental groups measured at indicated time points. 


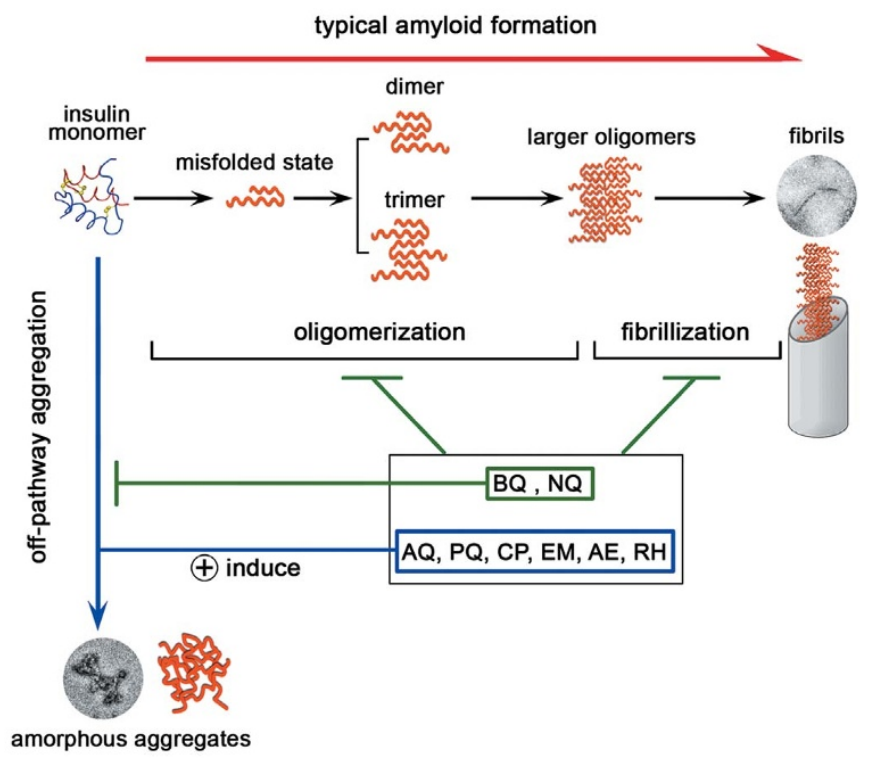

Figure $8 \mid$ A schematic representation of how quinones interfere the aggregation pathway of insulin.

fluorescence (a final ThT concentration of $20 \mu \mathrm{M}$ ) assay recorded on a Hitachi FL2700 fluorometer (Hitachi, Japan) at $482 \mathrm{~nm}$ with an excitation wavelength of $450 \mathrm{~nm}$ as we previously described ${ }^{59}$. The data were normalized against the DMSO or quinone controls background measured at the corresponding concentrations. For the aggregation intermediate disruption and fibril disaggregation experiments, each quinone was added to pre-incubated insulin at $9 \mathrm{~h}$ and $27 \mathrm{~h}$ respectively and the fluorescence was monitored in the following incubation. Each experiment was performed at least three times.

Far-UV circular dichroism (CD) and data analysis. A JASCO-810 circular dichroism spectropolarimeter (JASCO, Japan) was used to monitor secondary structures. Incubated samples were diluted to a final concentration of $20 \mu \mathrm{M}$ and detected in a $1 \mathrm{~mm}$ path length at $25^{\circ} \mathrm{C}$ under a constant flow of $\mathrm{N}_{2}$. CD spectra were obtained from 260 to $190 \mathrm{~nm}$ at a $50 \mathrm{~nm} / \mathrm{min}$ scanning speed and a $2 \mathrm{~nm}$ bandwidth. All samples were measured in triplicates and the averages were taken. The data were converted to mean residue ellipticity $[\theta]$ and were further calculated with the software $\mathrm{CDPro}^{60}$.

Size-exclusion chromatography (SEC). Incubated samples of designated time points were centrifuged and the supernatant was injected into a Tosoh TSK GW2000 column to perform SEC analysis on an L-2000 HPLC system (Hitachi, Japan). Elution buffer was $20 \%$ acetonitrile $(0.003 \%$ TFA) with a flow rate of $0.3 \mathrm{ml} / \mathrm{min}$ and a detection wavelength of $215 \mathrm{~nm}$.

Dynamic light scattering (DLS) analysis. The sizes of insulin aggregates were measured by dynamic light scattering in a zeta pals potential analyzer (Brookhaven Instruments, USA). Incubated samples were vortexed and detected at room temperature. The scattering angle was set at $90^{\circ}$. Each measurement was repeated three times and the average mean particle size was recorded. The data was analyzed by the multimodal size distribution (MSD) software as we previously described ${ }^{61}$.

Dot blot assay. Incubated samples were prepared as described. $3 \mu \mathrm{L}$ of sample solution aliquoted at indicated time points was blotted onto a nitrocellulose membrane (Biorad, USA). Dried membrane was blocked with 5\% non-fat milk for $1 \mathrm{~h}$ at room temperature and then incubated with A-11 (oligomer-specific antibody; $1: 2500$ ) or OC antibody (fibril-specific antibody; $1: 2500$ ) at $4{ }^{\circ} \mathrm{C}$ overnight. The membrane was incubated with anti-rabbit $\operatorname{IgG}(1: 5000)$ for $2 \mathrm{~h}$ at room temperature later. An ECL chemiluminescence kit (Advansta, USA) was used for the final blot development.

Transmission electronic microscopy (TEM). Samples for TEM assay were prepared as we previously described ${ }^{62}$. Incubated solution was applied onto a 300 -mesh formvar-carbon coated copper grid and sit for $5 \mathrm{~min}$. $1 \%$ freshly prepared uranyl formate was then dropwise added for staining. Dried samples were observed under a transmission microscope (Hitachi, Japan) operating at an accelerating voltage of $200 \mathrm{kV}$.

Hemolytic assay. Erythrocytes were separated from fresh blood through centrifugation at $1000 \mathrm{~g}$ and washed three times with isotonic phosphate buffered saline ( $\mathrm{pH} 7.4)$ as described ${ }^{63}$. Insulin with or without quinones were first incubated at $65^{\circ} \mathrm{C}$ for $24 \mathrm{~h}$, after the formation of aggregates were confirmed with the ThT assays, the incubated samples were mixed with cell suspensions (the final sample contains $2 \%$ hematocrit and $90 \mu \mathrm{M}$ insulin fibrils) and shaked at $200 \mathrm{rpm}$ for $24 \mathrm{~h}$ at $37^{\circ} \mathrm{C}$. To investigate the possible effects of quinones on fibril-induced membrane disruption, mature insulin fibrils were first mixed with erythrocytes, then quinones were added and incubated for another $24 \mathrm{~h}$. The absorption of supernatant was tested at $540 \mathrm{~nm}$. The hemolytic rate was calculated in relation to the hemolysis of erythrocytes with $1 \%$ Triton-100, which was taken as $100 \%$. Erythrocytes treated with DMSO and quinones, but without insulin were used as controls.

Statistical analysis. Each experiment was repeated three times and the data were expressed as the mean \pm SD. The Kruskal-Wallis test and the Mann-Whitney test were used to evaluate statistical significance. Difference was considered statistically significant at $\mathrm{P}<0.05$

1. Cao, P., Abedini, A. \& Raleigh, D. P. Aggregation of islet amyloid polypeptide: from physical chemistry to cell biology. Curr Opin Struct Biol 23, 82-9 (2013).

2. Dikiy, I. \& Eliezer, D. Folding and misfolding of alpha-synuclein on membranes. Biochim Biophys Acta 1818, 1013-8 (2012).

3. Nussbaum, J. M., Seward, M. E. \& Bloom, G. S. Alzheimer disease: a tale of two prions. Prion 7, 14-9 (2013).

4. Lopes, D. H. et al. Mechanism of islet amyloid polypeptide fibrillation at lipid interfaces studied by infrared reflection absorption spectroscopy. Biophys J 93, 3132-41 (2007).

5. Oren, Z. \& Shai, Y. Mode of action of linear amphipathic alpha-helical antimicrobial peptides. Biopolymers 47, 451-63 (1998).

6. Mattson, M. P. \& Goodman, Y. Different amyloidogenic peptides share a similar mechanism of neurotoxicity involving reactive oxygen species and calcium. Brain Res 676, 219-24 (1995).

7. Naiki, H. \& Nagai, Y. Molecular pathogenesis of protein misfolding diseases: pathological molecular environments versus quality control systems against misfolded proteins. J Biochem 146, 751-6 (2009).

8. Yumlu, S., Barany, R., Eriksson, M. \& Rocken, C. Localized insulin-derived amyloidosis in patients with diabetes mellitus: a case report. Hum Pathol 40, 1655-1660 (2009).

9. Okamura, S., Hayashino, Y., Kore-Eda, S. \& Tsujii, S. Localized Amyloidosis at the Site of Repeated Insulin Injection in a Patient With Type 2 Diabetes. Diabetes Care 36, E200-E200 (2013).

10. Brange, J., Andersen, L., Laursen, E. D., Meyn, G. \& Rasmussen, E. Toward understanding insulin fibrillation. J Pharm Sci 86, 517-25 (1997).

11. Huang, K., Dong, J., Phillips, N. B., Carey, P. R. \& Weiss, M. A. Proinsulin is refractory to protein fibrillation - Topological protection of a precursor protein from cross-beta assembly. Journal of Biological Chemistry 280, 42345-42355 (2005).

12. Seeliger, J. \& Winter, R. Islet amyloid polypeptide: aggregation and fibrillogenesis in vitro and its inhibition. Subcell Biochem 65, 185-209 (2012).

13. Eisele, Y. S. From soluble abeta to progressive abeta aggregation: could prion-like templated misfolding play a role? Brain Pathol 23, 333-41 (2013).

14. Marques, O. \& Outeiro, T. F. Alpha-synuclein: from secretion to dysfunction and death. Cell Death Dis 3, e350 (2012).

15. Pan, K. M. et al. Conversion of alpha-helices into beta-sheets features in the formation of the scrapie prion proteins. Proc Natl Acad Sci U S A 90, 10962-6 (1993).

16. Hill, S. E., Miti, T., Richmond, T. \& Muschol, M. Spatial Extent of Charge Repulsion Regulates Assembly Pathways for Lysozyme Amyloid Fibrils. PLoS One 6 (2011).

17. Nielsen, L., Frokjaer, S., Brange, J., Uversky, V. N. \& Fink, A. L. Probing the mechanism of insulin fibril formation with insulin mutants. Biochemistry $\mathbf{4 0}$ 8397-8409 (2001).

18. Nielsen, L., Frokjaer, S., Brange, J., Uversky, V. N. \& Fink, A. L. Probing the mechanism of insulin fibril formation with insulin mutants. Biochemistry 40 , 8397-409 (2001).

19. Wan, Z. L. et al. Diabetes-associated mutations in human insulin: crystal structure and photo-cross-linking studies of a-chain variant insulin Wakayama. Biochemistry 44, 5000-16 (2005).

20. Kachooei, E. et al. Inhibition study on insulin fibrillation and cytotoxicity by paclitaxel. J Biochem 155, 361-73 (2014).

21. Mishra, N. K., Joshi, K. B. \& Verma, S. Inhibition of human and bovine insulin fibril formation by designed peptide conjugates. Mol Pharm 10, 3903-12 (2013).

22. Cheng, B. et al. Inhibiting toxic aggregation of amyloidogenic proteins: a therapeutic strategy for protein misfolding diseases. Biochim Biophys Acta 1830, 4860-71 (2013).

23. Bieschke, J. et al. EGCG remodels mature alpha-synuclein and amyloid-beta fibrils and reduces cellular toxicity. Proc Natl Acad Sci US A 107, 7710-5 (2010).

24. Ono, K., Hasegawa, K., Naiki, H. \& Yamada, M. Curcumin has potent antiamyloidogenic effects for Alzheimer's beta-amyloid fibrils in vitro. J Neurosci Res 75, 742-50 (2004).

25. Mishra, R., Sellin, D., Radovan, D., Gohlke, A. \& Winter, R. Inhibiting islet amyloid polypeptide fibril formation by the red wine compound resveratrol. Chembiochem 10, 445-9 (2009).

26. Nie, Q., Du, X. G. \& Geng, M. Y. Small molecule inhibitors of amyloid beta peptide aggregation as a potential therapeutic strategy for Alzheimer's disease. Acta Pharmacol Sin 32, 545-51 (2011). 
27. Feng, S., Song, X. H. \& Zeng, C. M. Inhibition of amyloid fibrillation of lysozyme by phenolic compounds involves quinoprotein formation. FEBS Lett 586, 3951-5 (2012).

28. Convertino, M., Pellarin, R., Catto, M., Carotti, A. \& Caflisch, A. 9,10Anthraquinone hinders beta-aggregation: how does a small molecule interfere with Abeta-peptide amyloid fibrillation? Protein Sci 18, 792-800 (2009).

29. Bermejo-Bescos, P. et al. In vitro antiamyloidogenic properties of 1,4naphthoquinones. Biochem Biophys Res Commun 400, 169-74 (2010).

30. Misiti, F. et al. Protective effect of rhubarb derivatives on amyloid beta (1-42) peptide-induced apoptosis in IMR-32 cells: a case of nutrigenomic. Brain Res Bull 71, 29-36 (2006)

31. Liu, J. et al. Rhein lysinate decreases the generation of beta-amyloid in the brain tissues of Alzheimer's disease model mice by inhibiting inflammatory response and oxidative stress. J Asian Nat Prod Res 15, 756-63 (2013).

32. Pickhardt, M. et al. Anthraquinones inhibit tau aggregation and dissolve Alzheimer's paired helical filaments in vitro and in cells. J Biol Chem 280, 3628-35 (2005).

33. Siripong, P. et al. Antitumor activity of liposomal naphthoquinone esters isolated from Thai medicinal plant: Rhinacanthus nasutus KURZ. Biol Pharm Bull 29, 2279-83 (2006).

34. Dey, D., Ray, R. \& Hazra, B. Antitubercular and Antibacterial Activity of Quinonoid Natural Products Against Multi-Drug Resistant Clinical Isolates. Phytother Res (2013).

35. Kim, J. et al. Pyrroloquinoline quinone inhibits the fibrillation of amyloid proteins. Prion 4, 26-31 (2010).

36. Arora, A., Ha, C. \& Park, C. B. Insulin amyloid fibrillation at above 100 degrees C: new insights into protein folding under extreme temperatures. Protein Sci 13, 2429-36 (2004).

37. Dobson, C. M. Protein misfolding, evolution and disease. Trends Biochem Sci 24, 329-32 (1999).

38. Wang, J. B., Wang, Y. M. \& Zeng, C. M. Quercetin inhibits amyloid fibrillation of bovine insulin and destabilizes preformed fibrils. Biochem Biophys Res Commun 415, 675-9 (2011).

39. Grudzielanek, S. et al. Cytotoxicity of insulin within its self-assembly and amyloidogenic pathways. J Mol Biol 370, 372-84 (2007).

40. Kelly, J. W. Alternative conformations of amyloidogenic proteins govern their behavior. Curr Opin Struct Biol 6, 11-7 (1996).

41. Gillam, J. E. \& MacPhee, C. E. Modelling amyloid fibril formation kinetics: mechanisms of nucleation and growth. J Phys Condens Matter 25, 373101 (2013).

42. Uversky, V. N. Mysterious oligomerization of the amyloidogenic proteins. FEBS J 277, 2940-53 (2010).

43. Ehrnhoefer, D. E. et al. EGCG redirects amyloidogenic polypeptides into unstructured, off-pathway oligomers. Nat Struct Mol Biol 15, 558-66 (2008).

44. Zhang, T., Zhang, J., Derreumaux, P. \& Mu, Y. Molecular mechanism of the inhibition of EGCG on the Alzheimer Abeta(1-42) dimer. J Phys Chem B 117, 3993-4002 (2013).

45. Brender, J. R. et al. Zinc stabilization of prefibrillar oligomers of human islet amyloid polypeptide. Chem Commun (Camb) 49, 3339-41 (2013).

46. Hyung, S. J. et al. Insights into antiamyloidogenic properties of the green tea extract (-)-epigallocatechin-3-gallate toward metal-associated amyloid-beta species. Proc Natl Acad Sci U S A 110, 3743-3748 (2013).

47. Falconi, M. et al. Spectroscopic and molecular dynamics simulation studies of the interaction of insulin with glucose. Int J Biol Macromol 29, 161-8 (2001).

48. Ahmad, E. et al. A mechanistic approach for islet amyloid polypeptide aggregation to develop anti-amyloidogenic agents for type-2 diabetes. Biochimie 93, 793-805 (2011).

49. Wu, C., Lei, H., Wang, Z., Zhang, W. \& Duan, Y. Phenol red interacts with the protofibril-like oligomers of an amyloidogenic hexapeptide NFGAIL through both hydrophobic and aromatic contacts. Biophys J 91, 3664-72 (2006).

50. Meyer, E. A., Castellano, R. K. \& Diederich, F. Interactions with aromatic rings in chemical and biological recognition. Angew Chem Int Ed Engl 42, 1210-50 (2003).

51. Levy-Sakin, M., Shreberk, M., Daniel, Y. \& Gazit, E. Targeting insulin amyloid assembly by small aromatic molecules: toward rational design of aggregation inhibitors. Islets 1, 210-5 (2009).

52. DeToma, A. S., Salamekh, S., Ramamoorthy, A. \& Lim, M. H. Misfolded proteins in Alzheimer's disease and type II diabetes. Chem Soc Rev 41, 608-21 (2012).
53. Savelieff, M. G., Lee, S., Liu, Y. \& Lim, M. H. Untangling amyloid-beta, tau, and metals in Alzheimer's disease. ACS Chem Biol 8, 856-65 (2013).

54. Mancino, A. M., Hindo, S. S., Kochi, A. \& Lim, M. H. Effects of clioquinol on metal-triggered amyloid-beta aggregation revisited. Inorg Chem 48, 9596-8 (2009).

55. Hindo, S. S. et al. Small molecule modulators of copper-induced Abeta aggregation. J Am Chem Soc 131, 16663-5 (2009).

56. Gulaboski, R. et al. Hydroxylated derivatives of dimethoxy-1,4-benzoquinone as redox switchable earth-alkaline metal ligands and radical scavengers. Scientific Reports 3 (2013).

57. Yang, S. G. et al. alpha-Tocopherol quinone inhibits beta-amyloid aggregation and cytotoxicity, disaggregates preformed fibrils and decreases the production of reactive oxygen species, NO and inflammatory cytokines. Neurochem Int 57, 914-22 (2010).

58. Lepoittevin, J. P. \& Benezra, C. Allergic contact dermatitis caused by naturally occurring quinones. Pharm Weekbl Sci 13, 119-22 (1991).

59. Zhang, X. et al. Porcine islet amyloid polypeptide fragments are refractory to amyloid formation. FEBS Lett 585, 71-7 (2011).

60. Huang, K., Maiti, N. C., Phillips, N. B., Carey, P. R. \& Weiss, M. A. Structurespecific effects of protein topology on cross-beta assembly: studies of insulin fibrillation. Biochemistry 45, 10278-93 (2006).

61. Gong, H. et al. Bisphenol A accelerates toxic amyloid formation of human islet amyloid polypeptide: a possible link between bisphenol A exposure and type 2 diabetes. PLoS One 8, e54198 (2013).

62. Cheng, B. et al. Silibinin inhibits the toxic aggregation of human islet amyloid polypeptide. Biochem Biophys Res Commun 419, 495-9 (2012).

63. Li, Y. et al. Dissecting the role of disulfide bonds on the amyloid formation of insulin. Biochem Biophys Res Commun 423, 373-8 (2012).

\section{Acknowledgments}

The authors thank the Analytical and Testing Center of Huazhong University of Science and Technology, and the Research Core Facility of College of Life Sciences, Wuhan University for technical supports. This work was supported by the National Basic Research Program of China (2012CB524901), the Natural Science Foundation of China (Nos 31271370, 81100687, 81172971 and 81222043), the Program for New Century Excellent Talents in University (NECT11-0170), the Municipal Key Technology Program of Wuhan (Wuhan Bureau of Science \& Technology, No. 201260523174), the Health Bureau of Wuhan (WX12B06) and the Natural Science Foundation of Hubei Province (2011BCB012 and 2013CFB359).

\section{Author contributions}

Study concept and design: H.G., L.Z. and K.H. Acquisition of data: H.G., Z.H., B.C., A.P., X.Z. and Y.S. Drafting the manuscript and critical revision: H.G., A.P., L.Z. and K.H. All authors have approved the final version of the paper.

\section{Additional information}

Supplementary information accompanies this paper at http://www.nature.com/ scientificreports

Competing financial interests: The authors declare no competing financial interests. How to cite this article: Gong, H. et al. Effects of several quinones on insulin aggregation. Sci. Rep. 4, 5648; DOI:10.1038/srep05648 (2014)

This work is licensed under a Creative Commons Attribution-NonCommercialNoDerivs 4.0 International License. The images or other third party material in this article are included in the article's Creative Commons license, unless indicated otherwise in the credit line; if the material is not included under the Creative Commons license, users will need to obtain permission from the license holder in order to reproduce the material. To view a copy of this license, visit http:// creativecommons.org/licenses/by-nc-nd/4.0/ 